\title{
Radiation Effects in Flow through Porous Medium over a Rotating Disk with Variable Fluid Properties
}

\author{
Shalini Jain and Shweta Bohra \\ Department of Mathematics \& Statistics, Manipal University Jaipur, Jaipur 303007, India \\ Correspondence should be addressed to Shalini Jain; shalini.jain@jaipur.manipal.edu
}

Received 30 June 2016; Revised 3 September 2016; Accepted 15 September 2016

Academic Editor: Ali Cemal Benim

Copyright ( 92016 S. Jain and S. Bohra. This is an open access article distributed under the Creative Commons Attribution License, which permits unrestricted use, distribution, and reproduction in any medium, provided the original work is properly cited.

\begin{abstract}
The present study investigates the radiation effects in flow through porous medium over a permeable rotating disk with velocity slip and temperature jump. Fluid properties density $(\rho)$, viscosity $(\mu)$, and thermal conductivity $(\kappa)$ are taken to be dependent on temperature. Particular case considering these fluid properties' constant is also discussed. The governing partial differential equations are converted into nonlinear normal differential equation using similarity alterations. Transformed system of equations is solved numerically by using Runge-Kutta method with shooting technique. Effects of various parameters such as porosity parameter $K$, suction parameter $W_{s}$, rotational Reynolds number Re, Knudsen number Kn, Prandtl number Pr, radiation parameter $N$, and relative temperature difference parameter $\varepsilon$ on velocity profiles along radial, tangential, and axial direction and temperature distribution are investigated for both variable fluid properties and constant fluid properties. Results obtained are analyzed and depicted through graphs and table.
\end{abstract}

\section{Introduction}

The study of rotating disk flows of electrically conducting fluids has practical applications in many areas, such as rotating machinery, lubrication, computer storage devices, oceanography, viscometry, and crystal growth processes. In 1921, Kármán [1] was the first to investigate fluid flow due to a rotating disk. He introduced similarity transformations to transform governing partial differential equations into ordinary differential equations. Further Cochran [2], Benton [3], and Turkyilmazoglu [4] extended the work [1] and investigated flow and heat transfer under different boundary conditions. Chauhan and Gupta [5] investigated steady flow and heat transfer between two stationary naturally permeable disks. Heat transfer from a rotating disk by convection has been investigated theoretically under different physical and thermal conditions by Wagner [6], Millsaps and Pohlhausen [7], Kreith and Taylor [8], H.-T. Lin and L.-K. Lin [9], and Verma and Chauhan [10]. Chauhan and Jain [11] studied flow between rotating disks; they considered highly permeable disk. Turkyilmazoglu [12, 13] investigated flow and heat transfer of nanofluid due to rotating disk and on a radially shrinking rotating disk in the presence of a uniform vertical magnetic field, respectively. The unsteady magnetohydrodynamic (MHD) squeezing flow between two parallel disks (which is filled with nanofluid) is considered by Azimi and Riazi [14]; they have used Galerkin optimal homotopy asymptotic method (GOHAM) to solve the problem. Several researchers [15-17] have also investigated unsteady fluid flow and heat transfer over permeable rotating disk, rotating porous disk, and infinite rotating disk under different boundary conditions.

Radiative effects have several applications in physics and engineering field. Radiative heat transfer phenomena are used in nuclear reactors, power generation system, and high temperature plasma on controlling heating factor in industries and in liquid metal fluids. Several researchers investigated the effects of radiation on convective flows. Mansour [18] and Hossain et al. [19] studied the effect of radiation on free convection of fluid from a vertical plate and porous vertical plate, respectively. Raptis and Perdikis [20] investigated the MHD free convection flow in the presence of thermal 
radiation. The investigation of the effect of radiation on mixed convection flow of an optically dense viscous incompressible fluid along a heated vertical flat plate with uniform free stream and uniform surface temperature has been done by Hossain and Takhar [21]. A. Devi and R. U. Devi [22] studied thermal radiation effect on MHD flow over a rotating infinite nonporous disk. She also investigated porous rotating disk with Hall effect.

In recent years, the slip flow regime has been widely studied and researchers have been concentrating on the analysis of microscale in microelectromechanical systems (MEMS) associated with the embodiment of velocity slip and temperature jump. Wang [23] examined the flow due to a stretching boundary with partial slip-an exact solution of the Navier-Stokes equations. Osalusi [24] studied the combined hydromagnetic and slip flow of a steady, laminar conducting viscous fluid in the presence of thermal radiation due to an impulsively started rotating porous disk with the variable fluid properties. Khidir [25] investigated the effects of viscous dissipation and ohmic heating on steady MHD convective flow due to porous rotating disk taking into account the variable fluid properties. Sparrow et al. [26] considered the fluid flow due to the rotation of a porous surfaced disk and employed a set of linear slip flow conditions. He observed that a substantial reduction in torque occurred as a result of surface slip. Rashidi and Freidooni Mehr [27] investigated effects of velocity slip and temperature jump on the flow over a porous rotating disk. The combined effects of temperature and velocity jump on the heat transfer, fluid flow, and entropy generation over a single rotating disk have been examined by Arikoglu et al. [28]. Hayat et al. [29] investigated MHD steady flow of viscous nanofluid due to a rotating disk with partial slip.

In most of the research, the fluid properties such as density $(\rho)$, viscosity $(\mu)$, and thermal conductivity $(\kappa)$ are assumed to be constant. However, these properties remain unaltered if and only if temperature remains the same or does not change rapidly. Therefore, to predict flow behavior accurately, it is essential to consider variable fluid properties. Zakerullah and Ackroyd [30] analyzed the laminar natural convection boundary layer flow on a horizontal circular disk with variable properties. Herwig [31] and Herwig and Klemp [32] have extended the work [30]. They have investigated effects of variable properties in a tube and concentric annuli, respectively, at constant heat flux. Further, Maleque and Sattar $[33,34]$ have investigated laminar convective flow, taking into account the variable properties, due to a porous rotating disk. They solved the problems numerically using Runge-Kutta method with shooting technique. This work was extended by Osalusi and Sibanda [35]. They have studied flow in the presence of magnetic field. Rahman [36] made a study on the slip flow with variable properties due to a porous rotating disk.

Rashidi et al. [37] obtained analytical solutions flow and heat transfer over a rotating disk in porous medium. Hussain et al. [38] obtained numerical solution of a disk rotating in

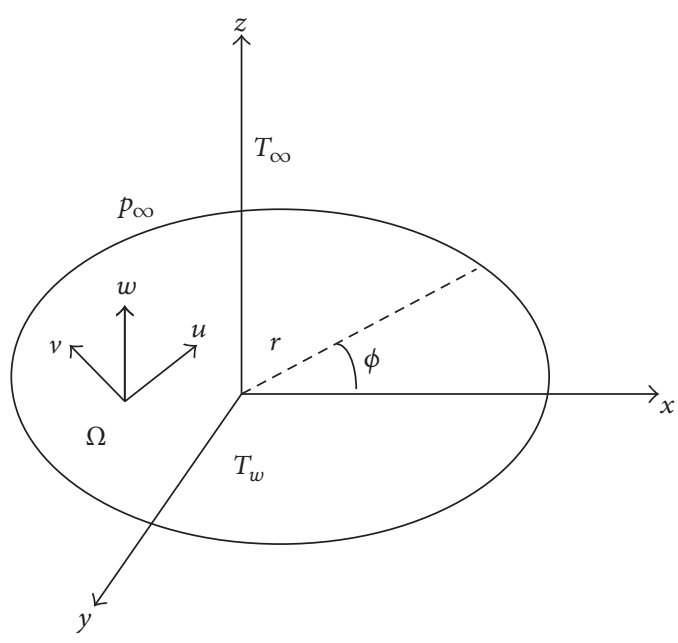

Figure 1: Coordinate system for the rotating disk flow.

a viscous fluid. Rashidi et al. $[39,40]$ analyzed entropy generation in a MHD flow over a rotating porous disk with variable physical properties. They have also investigated fluid flow over a permeable rotating disk in the presence of Soret and Dufour effect. Alam et al. [41] investigated thermophoretic deposition of micron sized particles on flow due to rotating disk.

The main goal of the present study is to investigate radiation effects in the steady flow over a rotating permeable disk in porous medium with velocity slip and temperature jump. To predict the flow behavior accurately variable thermophysical properties are taken into consideration. To the best of the author's knowledge, radiation effects of flow over rotating disk with velocity and temperature slip with variable thermal properties have not been studied yet. The novelty of present paper is to investigate flow and heat transfer for variable fluid properties with velocity slip and temperature jump, taken into consideration. Also, combined effects for both variable and constant fluid properties for various physical parameters on flow and heat transfer have been obtained and depicted graphically, which gives more insight about fluid flow (Figure 1).

\section{Mathematical Formulation}

Consider a steady slip flow due to permeable rotating disk through porous medium. Assume disk of $r$ radius is rotating with constant angular velocity $\Omega$ and placed at $z=0$ in cylindrical polar coordinates $(r, \phi, z)$, where $r$ and $\phi$ are the radial and tangential axis, respectively, and $z$ is the vertical axis.

Let $u, v$, and $w$ be the components of the fluid velocity in the direction of $r, \phi$, and $z$, respectively. $p$ and $T$ are the pressure and temperature of the fluid. The surface of the rotating disk is considered at a uniform temperature $T_{w}$. Pressure and temperature for ambient fluid are $p_{\infty}$ and $T_{\infty}\left(T_{w}>\right.$ $\left.T_{\infty}\right)$. 
Following [34, 35, 42], the fluid properties viscosity $(\mu)$, thermal conductivity $(\kappa)$, and the density $(\rho)$ are taken as functions of temperature:

$$
\begin{aligned}
& \mu=\mu_{\infty}\left(\frac{T}{T_{\infty}}\right)^{a}, \\
& \kappa=\kappa_{\infty}\left(\frac{T}{T_{\infty}}\right)^{b}, \\
& \rho=\rho_{\infty}\left(\frac{T}{T_{\infty}}\right)^{c},
\end{aligned}
$$

where $a, b$, and $c$ are arbitrary exponents and $\mu_{\infty}, \kappa_{\infty}$, and $\rho_{\infty}$ are the uniform viscosity, thermal conductivity, and density of the fluid. Assume $a=0.7, b=0.83$, and $c=-1.0$ (ideal gas) are the values of exponents for present investigation.

The governing equations of continuity, momentum, and energy for laminar incompressible flow in cylindrical coordinates are [35]

$$
\begin{aligned}
& \frac{\partial}{\partial r}(\rho r u)+\frac{\partial}{\partial z}(\rho r w)=0 \\
& \rho\left(u \frac{\partial u}{\partial r}+w \frac{\partial u}{\partial z}-\frac{v^{2}}{r}\right) \\
& =-\frac{\partial p}{\partial r}+\left[\frac{\partial}{\partial r}\left(\mu \frac{\partial u}{\partial r}\right)+\frac{\partial}{\partial r}\left(\mu \frac{u}{r}\right)+\frac{\partial}{\partial z}\left(\mu \frac{\partial u}{\partial z}\right)\right] \\
& -\frac{\mu u}{k}, \\
& \rho\left(u \frac{\partial v}{\partial r}+w \frac{\partial v}{\partial z}+\frac{u v}{r}\right) \\
& =\left[\frac{\partial}{\partial r}\left(\mu \frac{\partial v}{\partial r}\right)+\frac{\partial}{\partial r}\left(\mu \frac{v}{r}\right)+\frac{\partial}{\partial z}\left(\mu \frac{\partial v}{\partial z}\right)\right]-\frac{\mu v}{k} \\
& \rho\left(u \frac{\partial w}{\partial r}+w \frac{\partial w}{\partial z}\right) \\
& =-\frac{\partial p}{\partial r} \\
& +\left[\frac{\partial}{\partial r}\left(\mu \frac{\partial w}{\partial r}\right)+\frac{1}{r} \frac{\partial}{\partial r}(\mu w)+\frac{\partial}{\partial z}\left(\mu \frac{\partial w}{\partial z}\right)\right] \\
& \quad-\frac{\mu w}{k}, \\
& \rho C_{p}\left(u \frac{\partial T}{\partial r}+w \frac{\partial T}{\partial z}\right) \\
& \left.=\left[\kappa \frac{\partial T}{\partial r}\right)+\frac{\kappa}{r} \frac{\partial T}{\partial r}+\frac{\partial}{\partial z}\left(\kappa \frac{\partial T}{\partial z}\right)\right]-\frac{\partial q_{r}}{\partial z},
\end{aligned}
$$

where $k$ is the permeability of porous medium, $C_{p}$ is the specific heat at constant pressure, and $q_{r}$ is the radiative heat flux.

Subjected to the boundary conditions [27]

$$
\begin{aligned}
\text { at } z & =0 \\
u & =\frac{2-\sigma_{v}}{\sigma_{v}} \lambda \frac{\partial u}{\partial z}, \\
v & =r \Omega+\frac{2-\sigma_{v}}{\sigma_{v}} \lambda \frac{\partial v}{\partial z}, \\
w & =w_{0}, \\
T & =T_{w}+\frac{2-\sigma_{t}}{\sigma_{t}} \frac{2 \beta}{1+\beta} \frac{\lambda}{\operatorname{Pr}} \frac{\partial T_{s}}{\partial z}, \\
\text { at } z & \longrightarrow \infty \\
u & \longrightarrow \infty, \\
v & \longrightarrow \infty, \\
T & \longrightarrow T_{\infty},
\end{aligned}
$$

where $\sigma_{v}$ is the tangential momentum accommodation coefficient, $\sigma_{t}$ is the energy accommodation coefficient, $\lambda$ is mean free path, $\beta$ is the ratio of specific heats, and $T_{s}$ is the temperature of the fluid near to the disk surface.

Rosseland approximation has been used for radiation, $q_{r}$,

$$
q_{r}=-\frac{4 \sigma_{1} \partial T^{4}}{3 k_{1} \partial z}
$$

where $\sigma_{1}$ is the Stefan-Boltzmann constant and $T^{4}$ is the mean absorption coefficient. It is assumed that the temperature differences within the flow are sufficiently small so that the term $T^{4}$ may be expressed as a linear function of temperature. This is done by expanding $T^{4}$ in a Taylor series about $T_{\infty}$ and omitting the second- and higher-order terms leads to

$$
\begin{aligned}
T^{4} & \cong 4 T_{\infty}^{3} T-3 T_{\infty}^{4}, \\
\frac{\partial q_{r}}{\partial z} & =\frac{\partial}{\partial z}\left(\frac{-4 \sigma_{1}}{3 k_{1}} \frac{\partial T^{4}}{\partial z}\right) \\
& =\frac{\partial}{\partial z}\left(\frac{-4 \sigma_{1}}{3 k_{1}} \frac{\partial\left(4 T_{\infty}^{3} T-3 T_{\infty}^{4}\right)}{\partial z}\right) \\
& =\frac{-16 \sigma_{1} T_{\infty}^{3}}{3 k_{1}} \frac{\partial^{2} T}{\partial z^{2}} .
\end{aligned}
$$


The nondimensional form of the governing equations (2)-(6) is obtained by von-Kármán exact self-similar solution of the N-S equation:

$$
\begin{aligned}
\eta & =\left(\frac{\Omega}{v_{\infty}}\right)^{1 / 2} z, \\
u & =\Omega r F(\eta), \\
v & =\Omega r G(\eta), \\
w & =\left(\Omega \nu_{\infty}\right)^{1 / 2} H(\eta), \\
p-p_{\infty} & =-\mu_{\infty} \Omega P(\eta), \\
\theta(\eta) & =\frac{\left(T-T_{\infty}\right)}{\left(T_{w}-T_{\infty}\right)},
\end{aligned}
$$

where $v_{\infty}$ is uniform kinematic viscosity and $F, G, H, \theta$, and $P$ are nondimensional functions in terms of vertical coordinate $\eta$. Substituting (10) in (2)-(6), we get the system of following ordinary differential equations:

$$
\begin{aligned}
& H^{\prime}+2 F+c \varepsilon H \theta^{\prime}(1+\varepsilon \theta)^{-1}=0, \\
& F^{\prime \prime}-K F-(1+\varepsilon \theta)^{c-a}\left[F^{2}-G^{2}+H F^{\prime}\right] \\
& \quad+a \varepsilon(1+\varepsilon \theta)^{-1} \theta^{\prime} F^{\prime}=0, \\
& G^{\prime \prime}-K G-(1+\varepsilon \theta)^{c-a}\left[H G^{\prime}+2 F G\right] \\
& \quad+a \varepsilon(1+\varepsilon \theta)^{-1} \theta^{\prime} G^{\prime}=0, \\
& {\left[1+\frac{4 N}{3}(1+\varepsilon \theta)^{-b}\right] \theta^{\prime \prime}-\operatorname{Pr}(1+\varepsilon \theta)^{c-b} H \theta^{\prime}} \\
& \quad+b \varepsilon(1+\varepsilon \theta)^{-1} \theta^{\prime 2}=0,
\end{aligned}
$$

subject to boundary conditions

$$
\begin{aligned}
& \text { at } \eta=0 \\
& F(0)=\gamma F^{\prime}(0), \\
& G(0)=1+\gamma G^{\prime}(0), \\
& \theta(0)=1+\varphi \theta^{\prime}(0), \\
& H(0)=W_{s}, \\
& \text { at } \eta \longrightarrow \infty \\
& F(\eta) \longrightarrow 0, \\
& G(\eta) \longrightarrow 0, \\
& \theta(\eta) \longrightarrow 0,
\end{aligned}
$$

where $K=\nu_{\infty} / k \Omega, \operatorname{Pr}=\mu_{\infty} C_{p} / \kappa_{\infty}$, and $N=4 \sigma_{1} T_{\infty}^{3} / k_{1} \kappa_{\infty}$ are the porosity parameter, Prandtl number, and radiation parameter, respectively. $\varepsilon=\Delta T / T_{\infty}$ is the relative temperature difference parameter; it is positive for a heated surface, negative for a cooled surface and zero for the case of constant property.

$\mathrm{Kn}=\lambda / r$ is the Knudsen number; it is the ratio of mean free path of fluid particle diameter. For slip condition value ranges from 0.001 to 0.1 . For $\mathrm{Kn}<0.001$ no slip boundary conditions are valid; therefore velocity at the surface is zero. For high Knudsen number, high order continuum equation (Burnett equations) should be used [43]. For present investigation slip regime of Knudsen number which lies in the range $0.001<\mathrm{Kn}<0.1$ has been considered.

$\operatorname{Re}=\Omega r^{2} / \nu_{\infty}$ is the rotational Reynolds number; for laminar flow the value of the local Reynolds number is 1.8 $\times 10^{5}$ [44-46]. For transition flow the local Reynolds number values lie between $1.8 \times 10^{5}$ and $3.6 \times 10^{5}$ and for values higher than $3.6 \times 10^{5}$, the flow becomes turbulent. In this study the laminar flow for local Reynolds number that lies in the range $0<\operatorname{Re}<10000$ has been considered.

$\gamma=\left(\left(2-\sigma_{v}\right) / \sigma_{v}\right) \mathrm{Kn} \sqrt{\mathrm{Re}}, \gamma$ is the slip factor depending on rotational Reynolds number and Knudsen number and may vary from 0 to 12 .

$\varphi=\left(\left(2-\sigma_{t}\right) / \sigma_{t}\right)(2 \beta /(1+\beta))(\mathrm{Kn} / \operatorname{Pr}) \sqrt{\operatorname{Re}}, \varphi$ is the temperature jump factor, varying from 0 to 12 .

$W_{s}=w_{0} /\left(\Omega v_{\infty}\right)^{1 / 2}$ is the suction parameter, which has been taken as less than zero because suction of fluid is taking place.

The values of tangential momentum accommodation number $\left(\sigma_{v}\right)$, energy accommodation coefficient $\left(\sigma_{t}\right)$, and the specific heat ratio $(\beta)$ for air are considered as $0.9,0.9$, and 1.4 , respectively, Karniadakis et al. [47].

\section{Solution}

The nonlinear coupled ordinary differential equations (11) with the boundary conditions (12) have been solved numerically applying fourth-order Runge-Kutta scheme together with shooting method.

The given boundary value problem is reduced to the following system of initial value problem:

$$
\begin{aligned}
F^{\prime}= & p, \\
p^{\prime}= & K F+(1+\varepsilon \theta)^{c-a}\left[F^{2}-G^{2}+H p\right] \\
& -a \varepsilon(1+\varepsilon \theta)^{-1} s p, \\
G^{\prime}= & q, \\
q^{\prime}= & K G+(1+\varepsilon \theta)^{c-a}[2 F G+H q] \\
& -a \varepsilon(1+\varepsilon \theta)^{-1} s q, \\
H^{\prime}= & -2 F-c \varepsilon s H(1+\varepsilon \theta)^{-1}, \\
\theta^{\prime}= & s, \quad\left[1+4 N(1+\varepsilon \theta)^{-b} / 3\right] \\
s^{\prime}= & \frac{\operatorname{Pr}(1+\varepsilon \theta)^{c-b} H s-b \varepsilon(1+\varepsilon \theta)^{-1} s^{2}}{[1+} .
\end{aligned}
$$


Subject to boundary conditions (12) can be rewritten as

$$
\begin{aligned}
& F(0)=\gamma p(0), \\
& p(0)=r_{1}, \\
& G(0)=1+\gamma q(0), \\
& q(0)=r_{2}, \\
& H(0)=W_{s}, \\
& \theta(0)=1+\varphi s(0), \\
& s(0)=r_{3} .
\end{aligned}
$$

Particular Case. Assume fluid properties as constant.

From (1), we have

$$
\begin{aligned}
& \mu=\mu_{\infty}\left(\frac{T}{T_{\infty}}\right)^{a}=\mu_{\infty}\left(\frac{T_{\infty}+\Delta T \theta}{T_{\infty}}\right)^{a}, \\
& \mu=\mu_{\infty}\left(1+\frac{\Delta T \theta}{T_{\infty}}\right)=\mu_{\infty}\left[1+\left(\frac{\Delta T}{T_{\infty}}\right) \theta\right], \\
& \mu=\mu_{\infty}(1+\varepsilon \theta) .
\end{aligned}
$$

Taking $\varepsilon=0, \mu=\mu_{\infty}$ (constant).

Similarly at $\varepsilon=0, \rho=\rho_{\infty}$ and $\kappa=\kappa_{\infty}$.

Substitution of $\varepsilon=0$ in (2)-(4) and (6), system of equations is as follows:

$$
\begin{aligned}
H^{\prime}+2 F & =0, \\
F^{\prime \prime}-H F^{\prime}-F^{2}+G^{2}-K F & =0, \\
G^{\prime \prime}-H G^{\prime}-2 F G-K G & =0, \\
\left(1+\frac{4 N}{3}\right) \theta^{\prime \prime}-\operatorname{Pr} H \theta^{\prime} & =0 .
\end{aligned}
$$

Equations (16) under the boundary condition (12) are transformed into the following system of initial value problems:

$$
\begin{aligned}
F^{\prime} & =m, \\
m^{\prime} & =K F+\left(F^{2}-G^{2}+H m\right), \\
G^{\prime} & =n, \\
n^{\prime} & =K G+(2 F G+H n), \\
H^{\prime} & =-2 F \\
\theta^{\prime} & =t, \\
t^{\prime} & =\frac{t \operatorname{Pr} H}{(1+4 N / 3)} .
\end{aligned}
$$

The boundary conditions transformed as follows:

$$
\begin{aligned}
& F(0)=\gamma m(0), \\
& m(0)=r_{4}, \\
& G(0)=1+\gamma n(0), \\
& n(0)=r_{5}, \\
& H(0)=W_{s}, \\
& \theta(0)=1+\varphi t(0), \\
& t(0)=r_{6} .
\end{aligned}
$$

Here $r_{1}=F^{\prime}(0), r_{2}=G^{\prime}(0)$, and $r_{3}=\theta^{\prime}(0)$ are the initial guesses when fluid properties are variable and $r_{4}=F^{\prime}(0), r_{5}=$ $G^{\prime}(0)$, and $r_{6}=\theta^{\prime}(0)$ are the initial guesses when fluid properties are constant. The essence of present numerical method is to reduce the boundary value problem (BVP) into an initial value problem (IVP). Further, shooting technique is used to guess $r_{1}, r_{2}, r_{3}, r_{4}, r_{5}$, and $r_{6}$ until the boundary conditions are satisfied. A number of iterations of Runge-Kutta fourthorder method has been performed to obtain final values of these guesses. Initial guesses for different set of parameters are displayed in Table 1.

In this problem, the physical quantities of interest are local skin friction coefficients and the Nusselt number, which represents the wall shear stress and the rate of heat transfer, respectively. When variable fluid properties are taken into consideration, the fluid near to the disk opposes rotation of the disk, due to presence of tangential shear stress. Therefore, to maintain a steady rotation, it is essential to have torque at the shaft. The skin frictions $C f_{r}$ along radial direction and $C f_{t}$ along tangential direction, at no slip condition, are given as

$$
\begin{aligned}
& C f_{r}=\frac{\tau_{r}}{\rho U^{2}}, \\
& C f_{t}=\frac{\tau_{t}}{\rho U^{2}},
\end{aligned}
$$

where $U$ is linear velocity of disk,

$$
\begin{aligned}
& C f_{r}=\frac{\tau_{r}}{\rho_{\infty}(1+\varepsilon)^{b} \Omega^{2} r^{2}}, \\
& C f_{t}=\frac{\tau_{t}}{\rho_{\infty}(1+\varepsilon)^{b} \Omega^{2} r^{2}},
\end{aligned}
$$

and the Nusselt number $\mathrm{Nu}$ is given as

$$
\mathrm{Nu}=\frac{r q_{w}}{\kappa_{\infty}\left(T_{w}-T_{\infty}\right)}
$$


TABLE 1: Variation of $F^{\prime}(0),-G^{\prime}(0)$, and $-\theta^{\prime}(0)$ at the disk surface with $\varepsilon, K, \mathrm{Kn}, \mathrm{Re}, \operatorname{Pr}$, and $N$ parameters, when $W_{s}=-1$.

\begin{tabular}{|c|c|c|c|c|c|c|c|c|}
\hline$\varepsilon$ & K & $\mathrm{Kn}$ & $\mathrm{Re}$ & $\operatorname{Pr}$ & $N$ & $F^{\prime}(0)$ & $-G^{\prime}(0)$ & $-\theta^{\prime}(0)$ \\
\hline 0.2 & 1 & 0.05 & 100 & 1 & 1 & 0.031664472 & 0.775065344 & 0.294793960 \\
\hline 0.1 & & & & & & 0.031889982 & 0.796287340 & 0.316358091 \\
\hline \multirow[t]{17}{*}{0} & & & & & & 0.031640032 & 0.820381366 & 0.337225937 \\
\hline & 0 & & & & & 0.096135306 & 0.606295102 & 0.322588407 \\
\hline & 1 & & & & & 0.031664472 & 0.775065344 & 0.294793960 \\
\hline & 10 & & & & & 0.002466157 & 1.120998830 & 0.286444953 \\
\hline & & 0 & & & & 0.205650719 & 1.444447007 & 0.365643631 \\
\hline & & 0.02 & & & & 0.084184287 & 1.076778244 & 0.334910515 \\
\hline & & 0.05 & & & & 0.031664472 & 0.775065344 & 0.294793960 \\
\hline & & & 1 & & & 0.160224176 & 1.332713747 & 0.358173061 \\
\hline & & & 10 & & & 0.099394903 & 1.138256045 & 0.341296601 \\
\hline & & & 100 & 1 & & 0.031664472 & 0.775065344 & 0.294793960 \\
\hline & & & & 2 & & 0.032372329 & 0.772446756 & 0.576107763 \\
\hline & & & & 3 & & 0.032943882 & 0.770489197 & 0.860165056 \\
\hline & & & & 4 & & 0.033401361 & 0.768923963 & 1.143141070 \\
\hline & & & & & 0 & 0.032501654 & 0.780868622 & 0.521797333 \\
\hline & & & & & 1 & 0.031664472 & 0.775065344 & 0.294793960 \\
\hline & & & & & 2 & 0.031322694 & 0.773378512 & 0.217716214 \\
\hline & & & & & 3 & 0.031155065 & 0.772635266 & 0.181723226 \\
\hline
\end{tabular}

where radial shear stress $\tau_{r}$ and tangential shear stress $\tau_{t}$ are defined as

$$
\begin{aligned}
\tau_{r} & =\left[\mu\left(\frac{\partial u}{\partial z}+\frac{\partial w}{\partial r}\right)\right]_{z=0} \\
& =\mu_{\infty}(1+\varepsilon)^{a} \operatorname{Re}^{1 / 2} \Omega F^{\prime}(0), \\
\tau_{t} & =\left[\mu\left(\frac{\partial v}{\partial z}+\frac{1}{r} \frac{\partial w}{\partial \phi}\right)\right]_{z=0} \\
& =\mu_{\infty}(1+\varepsilon)^{a} \operatorname{Re}^{1 / 2} \Omega G^{\prime}(0)
\end{aligned}
$$

and using Fourier's law for rate of heat transfer $q_{w}$ is defined as

$$
\begin{aligned}
q_{w} & =-\left(\kappa \frac{\partial T}{\partial z}\right)_{z=0} \\
& =-\kappa_{\infty} \Delta T(1+\varepsilon)^{b}\left(\frac{\Omega}{\nu_{\infty}}\right)^{1 / 2} \theta^{\prime}(0)+q_{r}, \\
q_{w} & =\kappa_{\infty} \Delta T(1+\varepsilon)^{b}\left(\frac{\Omega}{v_{\infty}}\right)^{1 / 2}\left[1+\frac{4 N}{3}\right]\left[-\theta^{\prime}(0)\right] .
\end{aligned}
$$

Substituting (22) and (23) in (21), the radial and tangential skin frictions coefficients and Nusselt number are, respectively, given as

$$
\begin{aligned}
& (1+\varepsilon)^{c-a} \operatorname{Re}^{1 / 2} C f_{r}=F^{\prime}(0), \\
& (1+\varepsilon)^{c-a} \operatorname{Re}^{1 / 2} C f_{t}=G^{\prime}(0), \\
& (1+\varepsilon)^{-b} \operatorname{Re}^{-1 / 2} \mathrm{Nu}=-\left(1+\frac{4 N}{3}\right) \theta^{\prime}(0),
\end{aligned}
$$

where $\operatorname{Re}=\Omega r^{2} / \nu_{\infty}$ is rotational Reynolds number.

Thus (24) shows that the radial and tangential skin frictions coefficients are proportional to $F^{\prime}(0)$ and $G^{\prime}(0)$, respectively, and Nusselt number is proportional to $-\theta^{\prime}(0)$.

\section{Result and Discussion}

In this investigation Figures 2-6 show the effect of various values of the physical parameters on the velocity (radial, tangential, and axial) and temperature distribution. Results obtained, for both constant fluid property and variable fluid property, have been presented graphically.

Figures 2(a)-2(d) show the effect of porosity parameter $K$ on all velocity components and temperature distribution. It is observed that radial, tangential, and axial velocity decreases but temperature increases by increasing the permeability for both cases. Whereas variation in porosity parameter has less effect on the temperature distribution radial velocity attains 


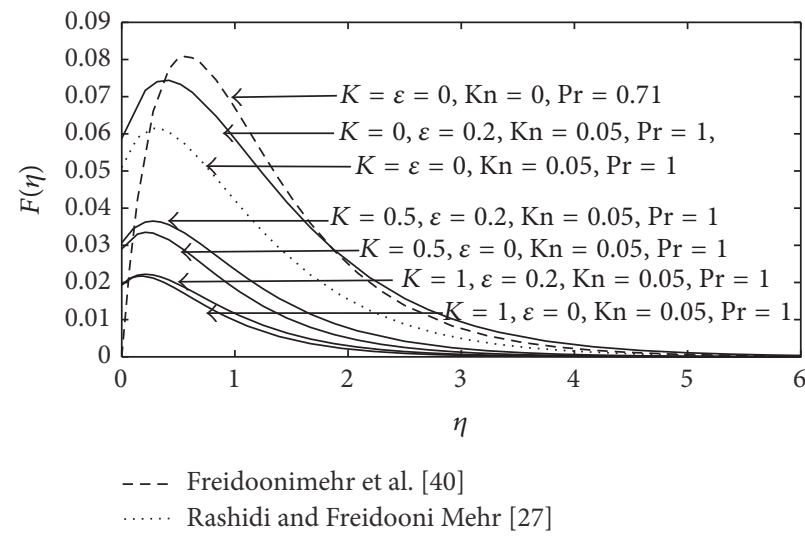

(a)

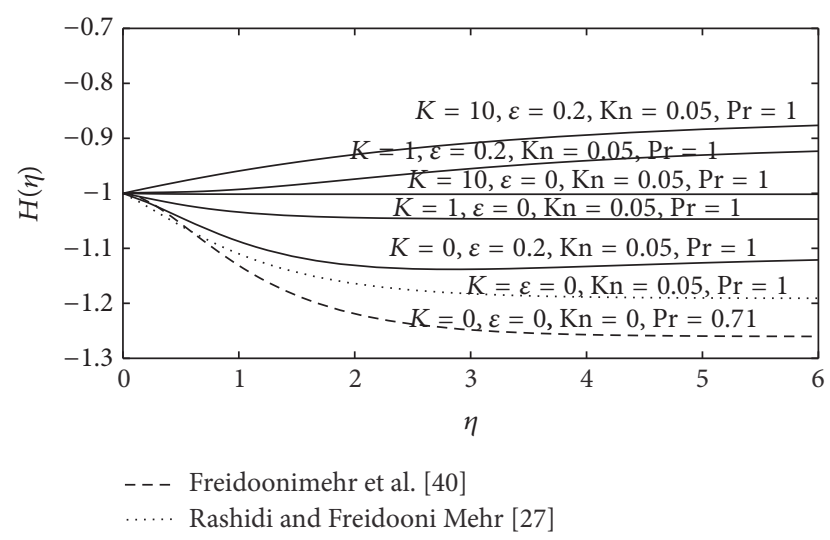

(c)

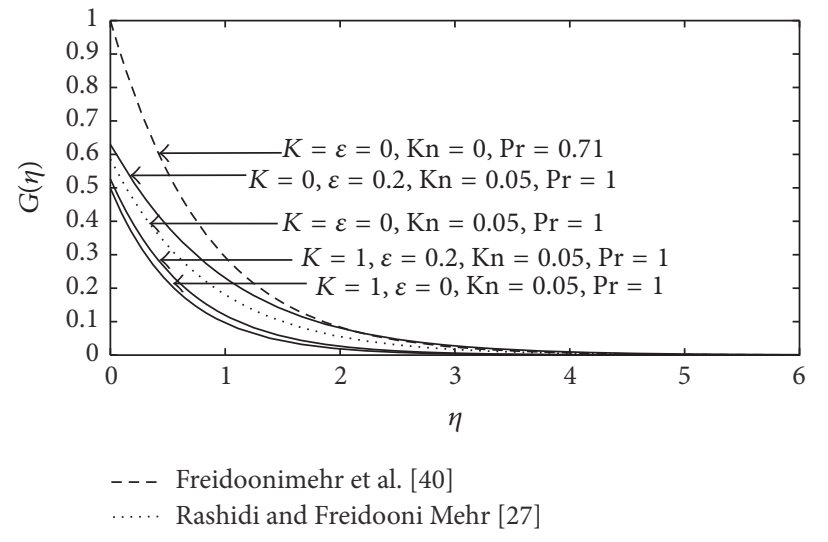

(b)

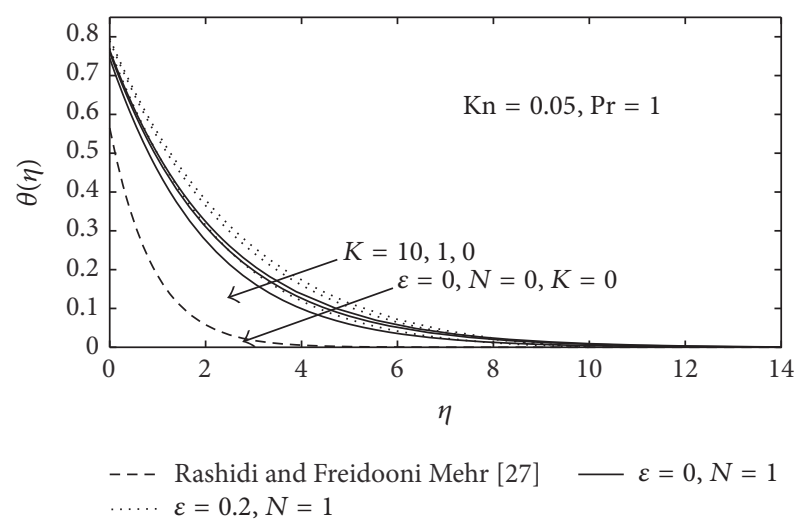

(d)

FiguRE 2: Effect of variation in the porosity parameter on the (a) radial, (b) tangential, (c) axial, and (d) temperature velocity profiles when $W_{s}=-1$ and $\operatorname{Re}=100$.

a maximum value close to the disk surface. When we reduce the present problem into the literature available by taking permeability parameter $K=0$ and radiation parameter $N=0$, then the result obtained is exactly similar to that of the Rashidi and Freidooni Mehr [27] and Freidoonimehr et al. [40]. This validates the results obtained for present investigation.

Figures 3(a)-3(d) depict the effect of suction parameter $W_{s}$ on the radial, tangential, axial, and temperature distribution, respectively. Figures 3(a)-3(c) show that all velocity components decrease as suction increases. Physical significance is that as suction increases, adherence of the fluid with the wall increases and as a result boundary layer thickness decreases.

Figure 3(d) depicts the effect of suction parameter on temperature profile. It is observed that as suction increases temperature of fluid decreases, because suction cools the boundary layer regime; therefore suction is used for cooling the flow in several engineering processes including MHD power generators and nuclear energy processes.

The effect of Reynolds number Re and Knudsen number $\mathrm{Kn}$ on velocity and temperature distribution is plotted in Figures 4 and 5, respectively. Both the parameters are showing the same effects on slip boundary conditions. It is observed that the increasing values of the Reynolds and Knudsen numbers decrease the fluid velocity components and temperature distribution. The case when value of Kn lies between 0.001 and 0.1 corresponds to slip at the surface of the disk. As slip increases the quantity of the fluid that can stick on the disk decreases. Therefore circumferential velocity of the fluid reduces and causes reduction in centrifugal force. As a result inward axial velocity decreases. In other words, as slip gets stronger, flow of fluid drawn or pushed away along the velocity directions decreases the heat generation.

Figure 6 shows the effect of Prandtl number on temperature profile. As Prandtl number increases the thermal boundary layer thickness decreases. Physical significance is that Prandtl number precludes dispersal of heat in the fluid.

Figure 7 depicts the variation of temperature profile with radiation. It is observed that as radiation increases temperature of the fluid increases. Also radiation parameter increases temperature gradient near the surface of the disk.

Figure 8 demonstrates the comparison of both constant and fluid properties on flow over a rotating disk with slip and 


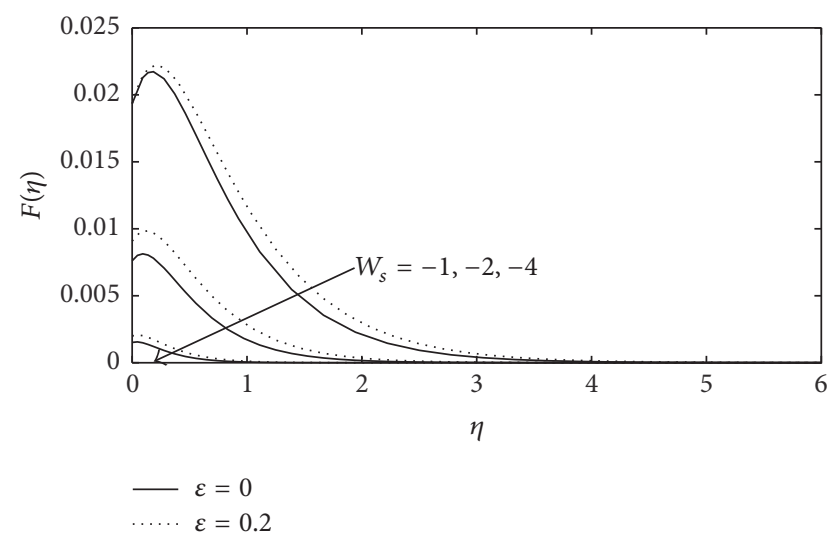

(a)

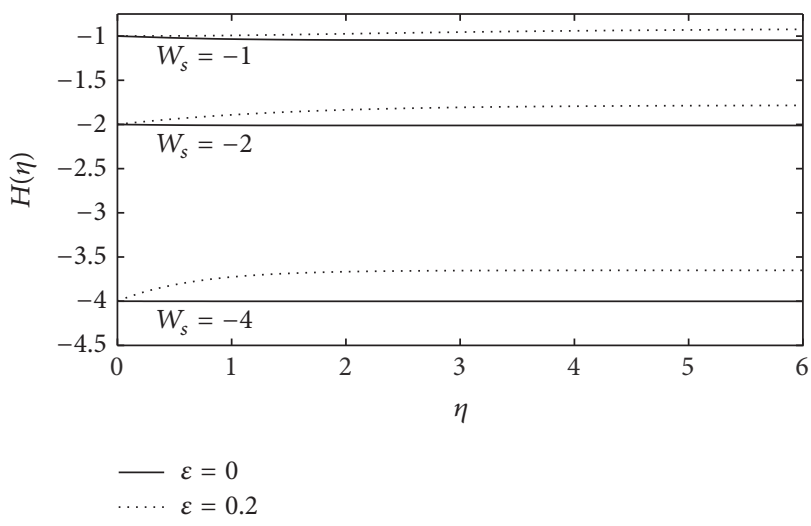

(c)

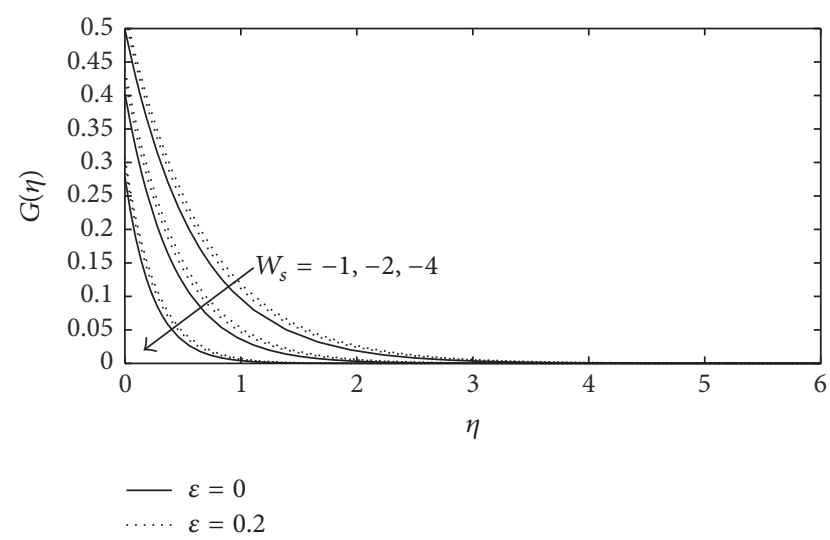

(b)

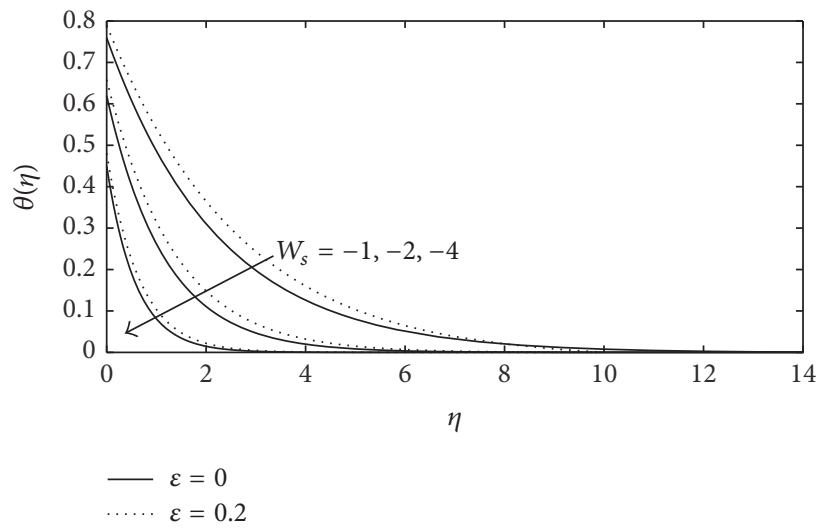

(d)

FIGURE 3: Effect of variation in the suction parameter on the (a) radial, (b) tangential, (c) axial, and (d) temperature velocity profiles when $K=1, \mathrm{Kn}=0.05, \operatorname{Re}=100, N=1$, and $\operatorname{Pr}=1$.

TABLE 2: Comparison between the results of present study with the results reported by Kelson and Desseaux [48], Maleque and Sattar [33], and Alam et al. [49] for the radial $F^{\prime}(0)$ and tangential $-G^{\prime}(0)$ skin friction coefficients for $\operatorname{Pr}=0.71, M=0, \varepsilon=0$, and $\mathrm{Kn}=0$.

\begin{tabular}{lcccccccc}
\hline \multirow{2}{*}{$W_{s}$} & \multicolumn{2}{c}{ Present } & \multicolumn{2}{c}{ Alam et al. [49] } & \multicolumn{2}{c}{ Maleque and Sattar [33] } & \multicolumn{2}{c}{ Kelson and Desseaux [48] } \\
& $F^{\prime}(0)$ & $-G^{\prime}(0)$ & $F^{\prime}(0)$ & $-G^{\prime}(0)$ & $F^{\prime}(0)$ & $-G^{\prime}(0)$ & $F^{\prime}(0)$ & $-G^{\prime}(0)$ \\
\hline 0 & 0.510213845 & 0.615909228 & 0.51022378 & 0.61592380 & 0.51015 & 0.61596 & 0.510233 & 0.615922 \\
-2 & 0.242412511 & 2.038595812 & 0.24241310 & 2.03859590 & 0.24251 & 2.03911 & 0.242421 & 2.038527 \\
-4 & 0.124738066 & 4.005180582 & 0.12475268 & 4.00526266 & 0.12477 & 4.00537 & 0.124742 & 4.005180 \\
-5 & 0.099914142 & 5.002660791 & 0.09991986 & 5.00271176 & 0.09996 & 5.00297 & 0.0999187 & 5.002661 \\
\hline
\end{tabular}

temperature jump. The radial velocity achieves a maximum value near to the surface of the disk for all values of $\varepsilon$. It is also noted that an increment in relative temperature difference parameter causes shifting of maximum point from the disk. The velocity along the tangential direction increases as value of relative temperature difference parameter increases whereas the velocity along axial direction decreases. Temperature increases as the value of relative temperature difference parameter increases. These results are similar to that obtained by Rashidi et al. [39].

Table 1 illustrates the effect of the parameters $\varepsilon, K$, $\mathrm{Kn}, \mathrm{Re}, \mathrm{Pr}$, and $N$ on constant suction parameter on the numerical values of $\left(F^{\prime}(0),-G^{\prime}(0)\right)$ and $\left(-\theta^{\prime}(0)\right)$. We observe that numerical value of $F^{\prime}(0),-G^{\prime}(0)$ and $-\theta^{\prime}(0)$ decreases with the increasing value of $\mathrm{Kn}, \mathrm{Re}$, and radiation parameter N.

Tables 2 and 3 depict the comparison of skin friction coefficients and rate of heat transfer between the results obtained in the present investigation and the literature available [33, $48,49]$. It is observed that results obtained in present study are very well in agreement with the existing results.

\section{Conclusions}

In this study, we have investigated radiation effect on velocity profile for all components and temperature profile through rotating disk in porous medium for variable fluid properties and in particular case for constant fluid properties also. By 

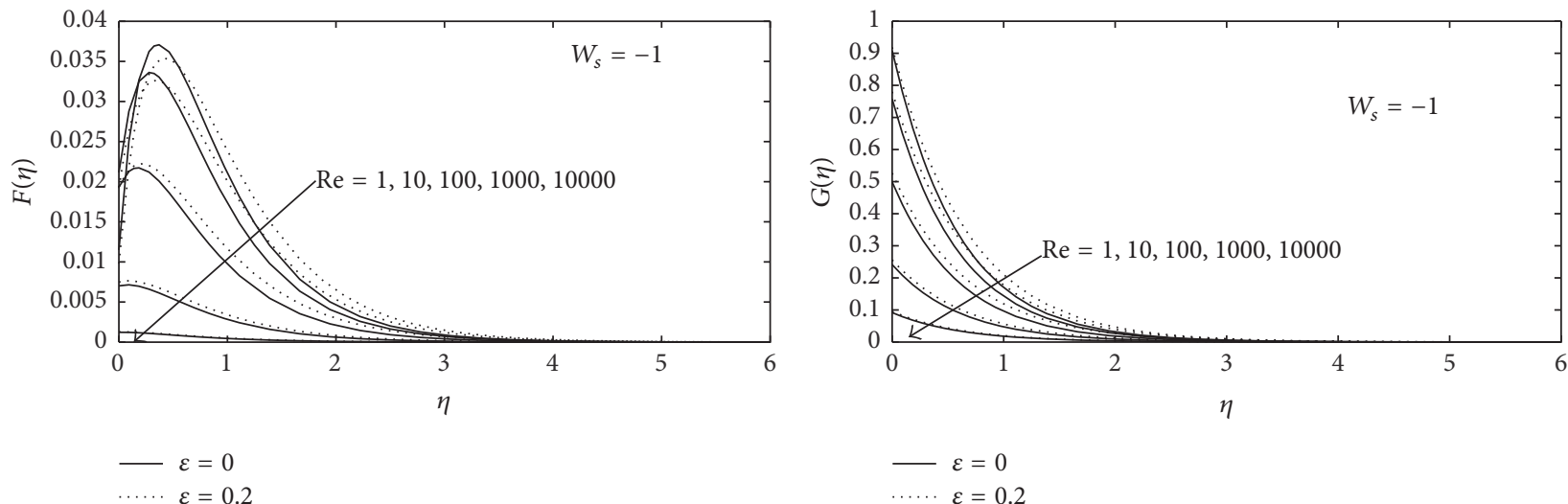

(a)

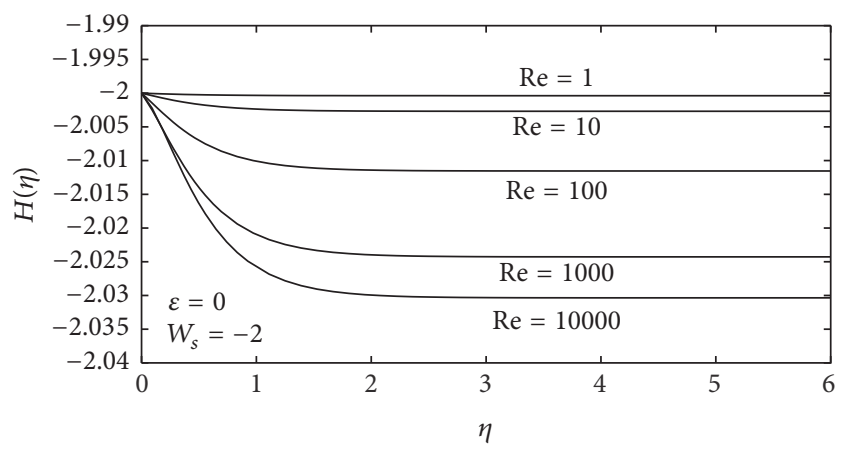

(c) (b)

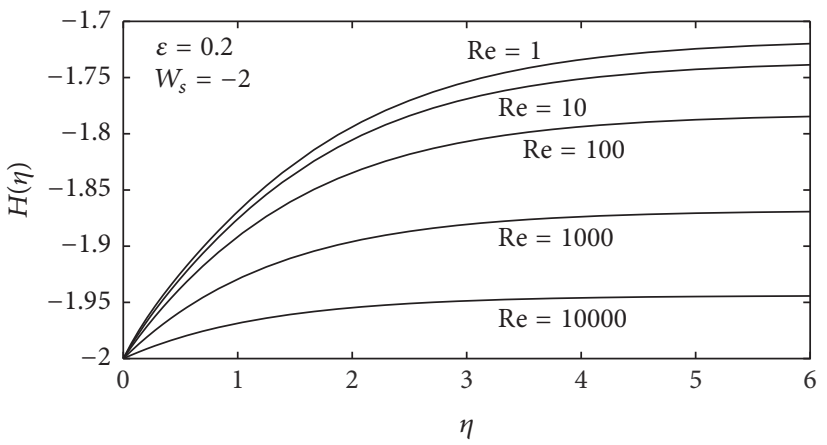

(d)

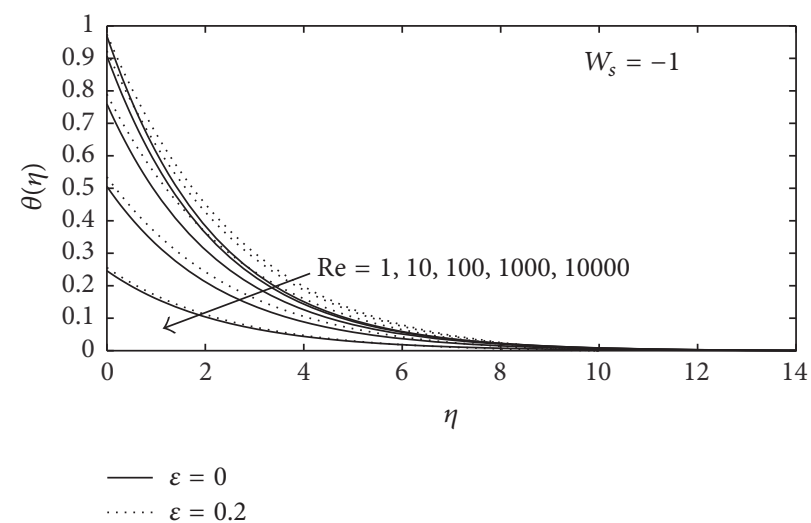

(e)

FIGURE 4: Effect of variation in the Reynolds number on the (a) radial, (b) tangential, (c) \& (d) axial, and (e) temperature velocity profiles when $K=1, \mathrm{Kn}=0.05, N=1$, and $\operatorname{Pr}=1$.

TABLE 3: Comparison between the results of present study with the results reported by Kelson and Desseaux [48], Maleque and Sattar [33], and Alam et al. [49] for rate of heat transfer $-\theta^{\prime}(0)$ for $\operatorname{Pr}=0.71, M=0, \varepsilon=0, \mathrm{Kn}=0$, and $N=0$.

\begin{tabular}{lcccc}
\hline$W_{s}$ & Present & Alam et al. [49] & Maleque and Sattar [33] & $-\begin{array}{c}\text { Kelson and Desseaux [48] } \\
-\theta^{\prime}(0)\end{array}$ \\
\hline 0 & $-\theta^{\prime}(0)$ & $-\theta^{\prime}(0)$ & 0.32576 & 0.325856 \\
-2 & 0.326798372 & 0.32637889 & 1.44212 & 1.437782 \\
-4 & 1.438764651 & 1.43876482 & 2.84470 & 2.842381 \\
-5 & 2.842381877 & 2.84369011 & 3.55411 & 3.551223 \\
\hline
\end{tabular}




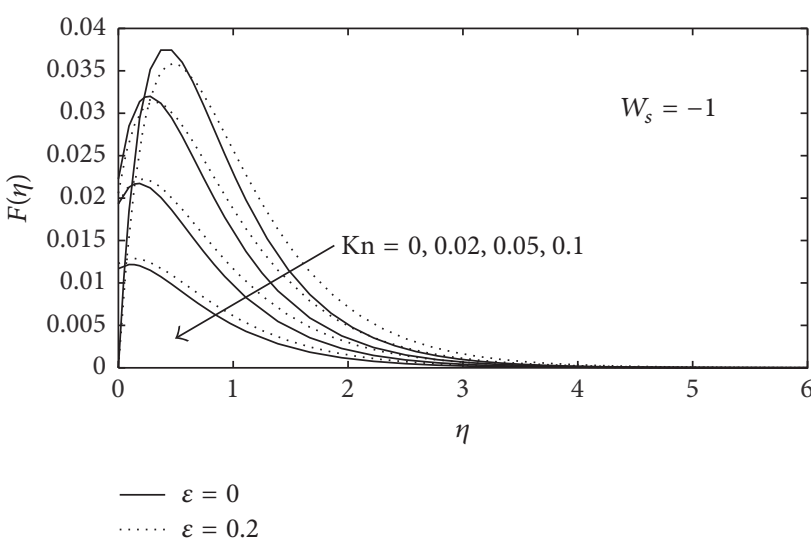

(a)

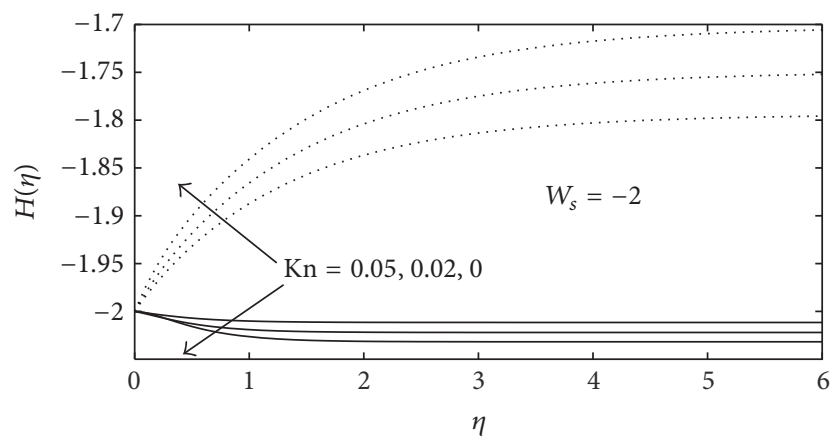

$\begin{aligned} \varepsilon & =0 \\ \varepsilon & =0.2\end{aligned}$

(c)

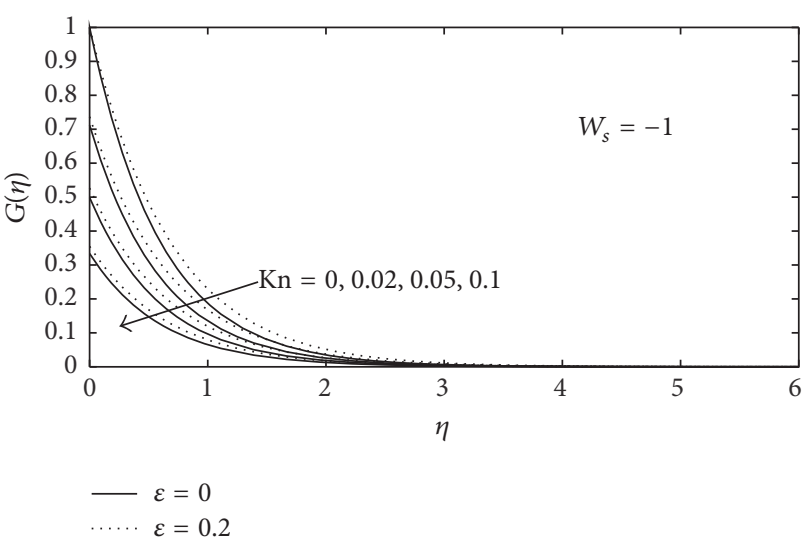

(b)

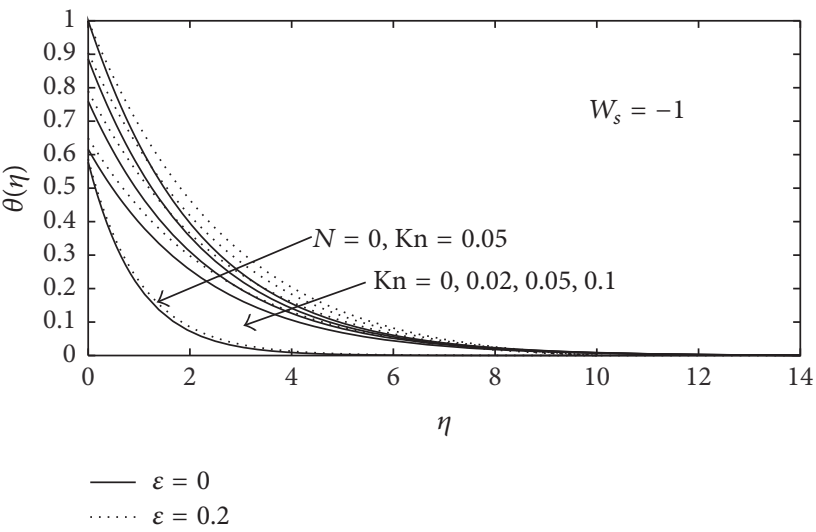

(d)

FIGURE 5: Effect of variation in the Knudsen number on the (a) radial, (b) tangential, (c) axial, and (d) temperature velocity profiles when $K=1, \operatorname{Re}=100, N=1$, and $\operatorname{Pr}=1$.

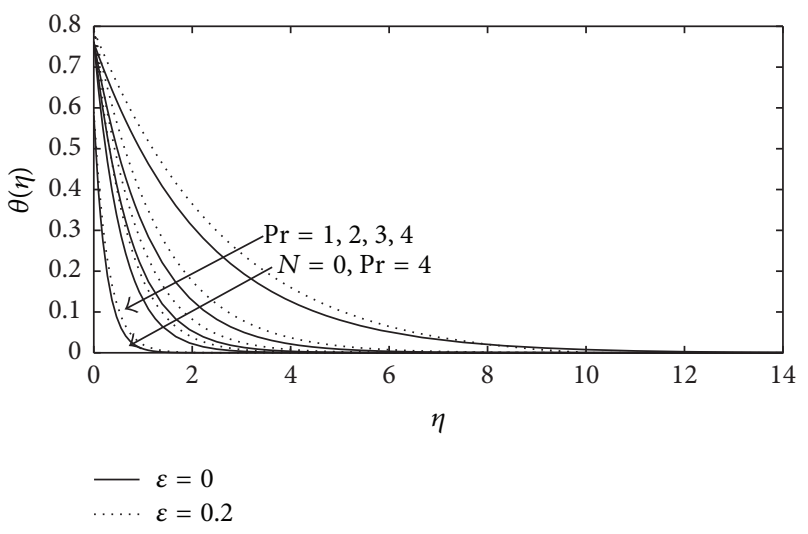

FIGURE 6: Effect of Prandtl number on temperature distribution when $K=1, W_{s}=-1, \operatorname{Re}=100, \mathrm{Kn}=0.05$, and $N=1$.

similarity transformation governing equations transformed into nonlinear ordinary differential equations which are solved numerically by using Runge-Kutta method with shooting technique. Based on the resulting solutions the following conclusions can be drawn:

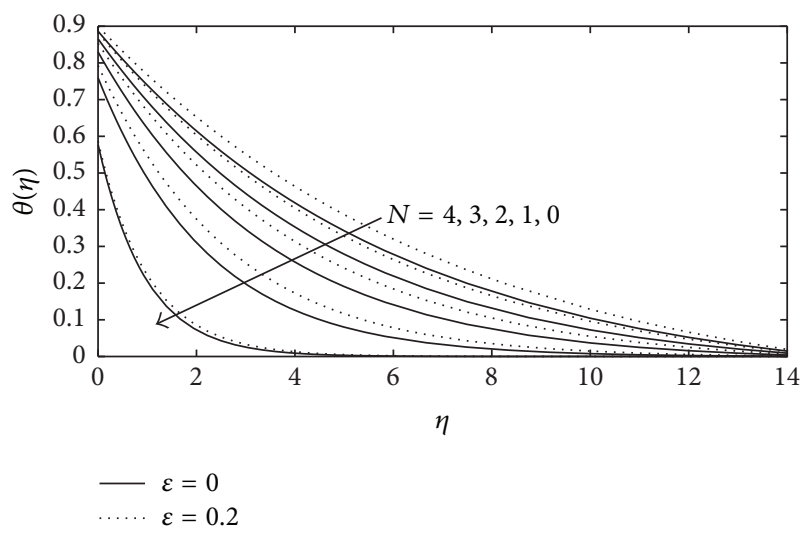

FIGURE 7: Effect of radiation parameter on temperature distribution when $K=1, W_{s}=-1, \operatorname{Re}=100, \mathrm{Kn}=0.05$, and $\operatorname{Pr}=1$.

(i) The radial, tangential, and axial velocity profiles decrease while the temperature increases with the increasing values of porosity parameter.

(ii) The increasing value of Reynolds and Knudsen number decreases the fluid velocity components and 


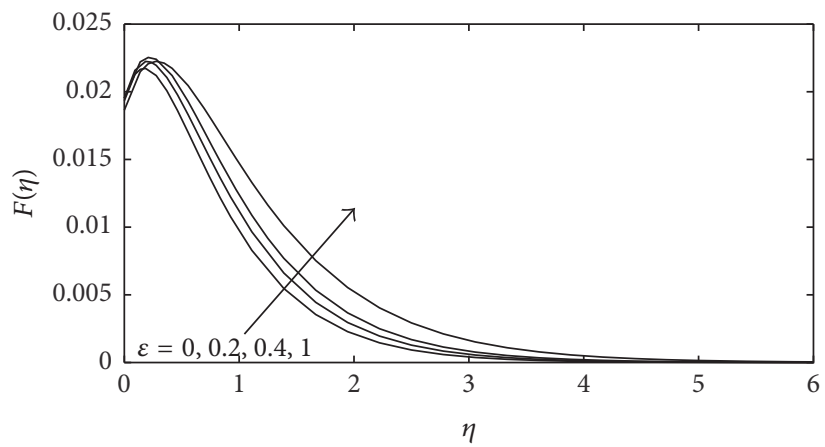

(a)

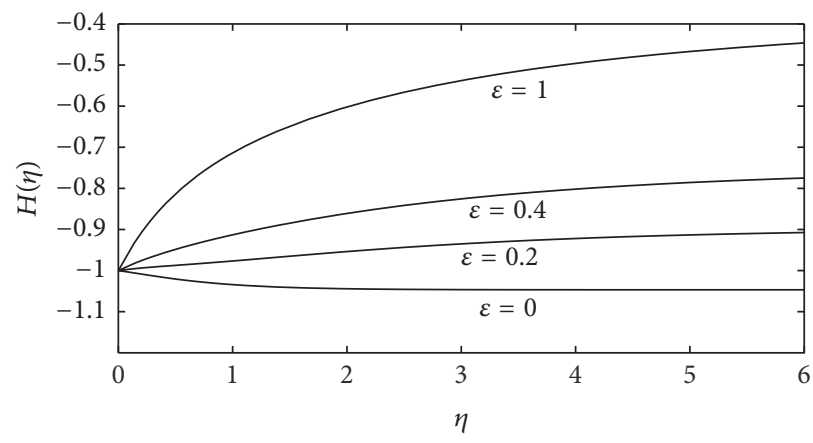

(c)

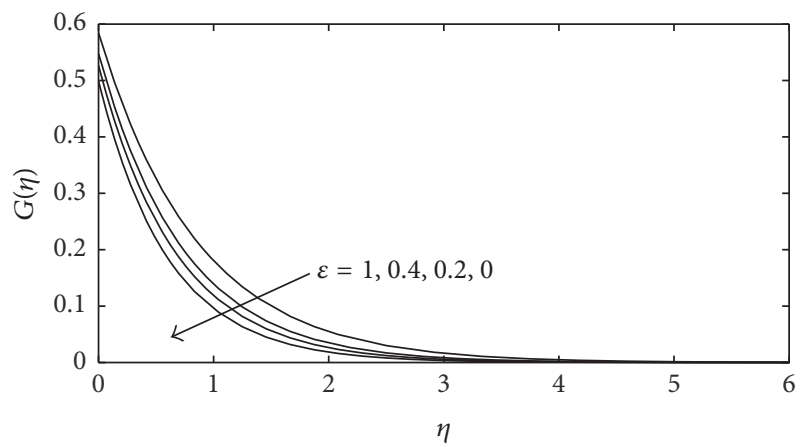

(b)

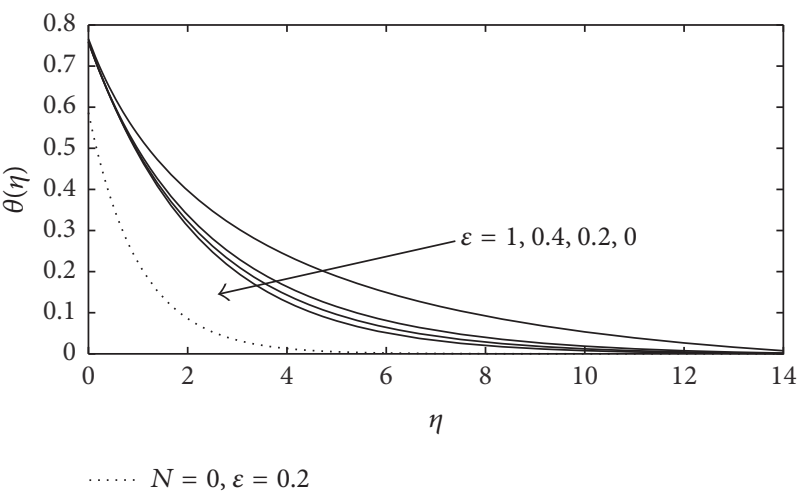

(d)

FIGURE 8: Effect of variation in the relative temperature difference parameter on the (a) radial, (b) tangential, (c) axial, and (d) temperature velocity profiles when $K=1, W_{s}=-1, \mathrm{Kn}=0.05, \mathrm{Re}=100, N=1$, and $\operatorname{Pr}=1$.

temperature and suction parameter also shows the same effect.

(iii) For the effect of the radiation parameter on the temperature distribution, it is seen that the temperature distribution decreases with the increasing values of radiation parameter and also it has been observed that the radial and tangential skin friction values decrease with increase in the radiation parameter.

\section{Competing Interests}

The authors declare that they have no competing interests.

\section{References}

[1] Th. V. Kármán, "Uber laminare und turbulente reibun," Zeitschrift für Angewandte Mathematik und Mechanik, vol. 1, no. 4, pp. 233-252, 1921.

[2] W. G. Cochran, "The flow due to a rotating disk," Mathematical Proceedings of the Cambridge Philosophical Society, vol. 30, no. 3, pp. 365-375, 1934.

[3] E. R. Benton, "On the flow due to a rotating disk," Journal of Fluid Mechanics, vol. 24, no. 4, pp. 781-800, 1966.

[4] M. Turkyilmazoglu, "Purely analytic solutions of the compressible boundary layer flow due to a porous rotating disk with heat transfer," Physics of Fluids, vol. 21, no. 10, Article ID 106104, 2009.
[5] D. S. Chauhan and S. Gupta, "Steady flow and heat transfer between two stationary naturally permeable disks," Journal of Ultra Scientist of Physical Sciences, vol. 12, no. 1, pp. 45-52, 2000.

[6] C. Wagner, "Heat transfer from a rotating disk to ambient air," Journal of Applied Physics, vol. 19, no. 9, pp. 837-839, 1948.

[7] K. Millsaps and K. Pohlhausen, "Heat transfer by laminar flow from a rotating plate," Journal of the Aeronautical Sciences, vol. 19, pp. 120-126, 1952.

[8] F. Kreith and J. H. Taylor, "Heat transfer from a rotating disk in turbulent flow, no. , 1956.," ASME Paper 56-A-146, 1956.

[9] H.-T. Lin and L.-K. Lin, "Heat transfer from a rotating cone or disk to fluids of any Prandtl number," International Communications in Heat and Mass Transfer, vol. 14, no. 3, pp. 323-332, 1987.

[10] P. D. Verma and D. S. Chauhan, "Flow between a torsionally oscillating impermeable disc and a stationary naturally permeable disc," Indian Journal of Pure and Applied Mathematics, vol. 10, pp. 1351-1361, 1979.

[11] D. S. Chauhan and S. Jain, "Steady flow between highly permeable rotating disks," Indian Journal of Theoretical Physics, vol. 52, no. 1, pp. 39-50, 2004.

[12] M. Turkyilmazoglu, "Nanofluid flow and heat transfer due to a rotating disk," Computers \& Fluids, vol. 94, pp. 139-146, 2014.

[13] M. Turkyilmazoglu, "MHD fluid flow and heat transfer due to a shrinking rotating disk," Computers \& Fluids, vol. 90, pp. 51-56, 2014.

[14] M. Azimi and R. Riazi, "Heat transfer analysis of GO-water nanofluid flow between two parallel disks," Propulsion and Power Research, vol. 4, no. 1, pp. 23-30, 2015. 
[15] B. R. Sharma and H. Konwar, "Effect of chemical reaction on mass transfer due to a permeable rotating heated disk," International Journal of Computer Applications, vol. 119, no. 21, pp. 5-9, 2015.

[16] H. Shahmohamadi and M. Mohammadpour, "A series solution for three-dimensional navier-stokes equations of flow near an infinite rotating disk," World Journal of Mechanics, vol. 4, pp. 117-127, 2014.

[17] S. Srinivas, A. S. Reddy, T. R. Ramamohan, and A. K. Shukla, "Thermal-diffusion and diffusion-thermo effects on MHD flow of viscous fluid between expanding or contracting rotating porous disks with viscous dissipation," Journal of the Egyptian Mathematical Society, vol. 24, no. 1, pp. 100-107, 2016.

[18] M. A. Mansour, "Radiative and free-convection effects on the oscillatory flow past a vertical plate," Astrophysics and Space Science, vol. 166, no. 2, pp. 269-275, 1990.

[19] M. A. Hossain, M. A. Alim, and D. A. S. Rees, "The effect of radiation on free convection from a porous vertical plate," International Journal of Heat and Mass Transfer, vol. 42, no. 1, pp. 181-191, 1999.

[20] A. Raptis and C. Perdikis, "MHD free convection flow by the presence of radiation," International Journal of Magnetohydrodynamics Plasma and Space Research, vol. 9, pp. 237-252, 2000.

[21] M. A. Hossain and H. S. Takhar, "Radiation effect on mixed convection along a vertical plate with uniform surface temperature," Heat and Mass Transfer, vol. 31, no. 4, pp. 243-248, 1996.

[22] A. Devi and R. U. Devi, "Effects of thermal radiation on hydromagnetic flow due to a porous rotating disk with hall effect," Journal of Applied Fluid Mechanics, vol. 5, no. 2, pp. 17, 2012.

[23] C. Y. Wang, "Flow due to a stretching boundary with partial slip-an exact solution of the Navier-Stokes equations," Chemical Engineering Science, vol. 57, no. 17, pp. 3745-3747, 2002.

[24] E. Osalusi, "Effects of thermal radiation on MHD and slip flow over a porous rotating disk with variable properties," Romanian Journal of Physics, vol. 52, no. 3-4, pp. 217-229, 2007.

[25] A. A. Khidir, "Viscous dissipation, Ohmic heating and radiation effects on MHD flow past a rotating disk embedded in a porous medium with variable properties," Arabian Journal of Mathematics, vol. 2, no. 3, pp. 263-277, 2013.

[26] E. M. Sparrow, G. S. Beavers, and L. Y. Hung, "Flow about a porous-surfaced rotating disk," International Journal of Heat and Mass Transfer, vol. 14, no. 7, pp. 993-996, 1971.

[27] M. M. Rashidi and N. Freidooni Mehr, "Effects of velocity slip and temperature jump on the entropy generation in magnetohydrodynamic flow over a porous rotating disk," Journal of Mechanical Engineering, vol. 1, no. 3, 2012.

[28] A. Arikoglu, G. Komurgoz, I. Ozkol, and A. Y. Gunes, "Combined effects of temperature and velocity jump on the heat transfer, fluid flow, and entropy generation over a single rotating disk," Journal of Heat Transfer, vol. 132, no. 11, Article ID 111703, 2010.

[29] T. Hayat, M. Rashid, M. Imtiaz, and A. Alsaedi, "Magnetohydrodynamic (MHD) flow of $\mathrm{Cu}$-water nanofluid due to a rotating disk with partial slip," AIP Advances, vol. 5, no. 6, Article ID 067169, 2015.

[30] M. Zakerullah and J. A. D. Ackroyd, "Laminar natural convection boundary-layers on Horizontal Circular disks," Journal of Applied Mathematics and Physics, vol. 30, pp. 427-435, 1979.

[31] H. Herwig, "The effect of variable properties on momentum and heat transfer in a tube with constant heat flux across the wall," International Journal of Heat and Mass Transfer, vol. 28, no. 2, pp. 423-431, 1985.
[32] H. Herwig and K. Klemp, "Variable property effects of fully developed laminar flow in concentric annuli," Journal of Heat Transfer, vol. 110, no. 2, pp. 314-320, 1988.

[33] K. A. Maleque and M. A. Sattar, "Steady laminar convective flow with variable properties due to a porous rotating disk," Journal of Heat Transfer, vol. 127, no. 12, pp. 1406-1409, 2005.

[34] K. A. Maleque and M. A. Sattar, "The effects of variable properties and hall current on steady MHD laminar convective fluid flow due to a porous rotating disk," International Journal of Heat and Mass Transfer, vol. 48, no. 23-24, pp. 4963-4972, 2005.

[35] E. Osalusi and P. Sibanda, "On variable laminar convective flow properties due to a porous rotating disk in a magnetic field," Romanian Journal of Physics, vol. 9, no. 10, pp. 933-944, 2006.

[36] M. M. Rahman, "Convective Hydromagnetic slip flow with variable properties due to a porous rotating disk," The Sultan Qaboos University Journal for Science, vol. 15, pp. 55-79, 2010.

[37] M. M. Rashidi, S. A. Mohimanian Pour, T. Hayat, and S. Obaidat, "Analytic approximate solutions for steady flow over a rotating disk in porous medium with heat transfer by homotopy analysis method," Computers \& Fluids, vol. 54, no. 1, pp. 1-9, 2012 .

[38] S. Hussain, F. Ahmad, M. Shafique, and S. Hussain, "Numerical solution for accelerated rotating disk in a viscous fluid," Applied Mathematics, vol. 4, no. 6, pp. 899-902, 2013.

[39] M. M. Rashidi, N. Kavyani, and S. Abelman, "Investigation of entropy generation in MHD and slip flow over a rotating porous disk with variable properties," International Journal of Heat and Mass Transfer, vol. 70, pp. 892-917, 2014.

[40] N. Freidoonimehr, M. M. Rashidi, S. Abelman, and G. Lorenzini, "Analytical modeling of MHD flow over a permeable rotating disk in the presence of Soret and Dufour effects: entropy analysis," Entropy, vol. 18, no. 5, article 131, 2016.

[41] M. S. Alam, S. M. Chapal Hossain, and M. M. Rahman, "Transient thermophoretic particle deposition on forced convective heat and mass transfer flow due to a rotating disk," Ain Shams Engineering Journal, vol. 7, no. 1, pp. 441-452, 2016.

[42] S. Jayaraj, “Thermophoresis in laminar flow over cold inclined plates with variable properties," Heat and Mass Transfer, vol. 30, no. 3, pp. 167-173, 1995.

[43] M. M. Rahman, "Thermophoretic deposition of nanoparticles due to a permeable rotating disk: effects of partial slip, magnetic field, thermal radiation, thermal-diffusion, and diffusionthermo," International Journal of Mathematical, Computational, Physical, Electrical and Computer Engineering, vol. 7, no. 5, 2013.

[44] I. V. Shevchuk, Convective Heat and Mass Transfer in Rotating Disk Systems, Springer, Berlin, Germany, 2009.

[45] C. L. Tien and D. T. Campbell, "Heat and mass transfer from rotating cones," Journal of Fluid Mechanics, vol. 17, no. 1, pp. 105$112,1963$.

[46] C. J. Elkins and J. K. Eaton, "Heat transfer in the rotating disk boundary layer," Tech. Rep. TSD-103, Stanford University, Department of Mechanical Engineering, Thermosciences Division, Stanford, Calif, USA, 1997.

[47] G. Karniadakis, A. Beskok, and N. Aluru, Microflows: Fundamentals and Simulation, Springer, New York, NY, USA, 2001.

[48] N. Kelson and A. Desseaux, "Note on porous rotating disk flow," Australian \& New Zealand Industrial and Applied Mathematics Journal, vol. 42, pp. 837-855, 2000.

[49] M. Alam, N. Poddar, M. Rahman, and K. Vajravelu, "Transient hydromagnetic forced convective heat transfer slip flow due to a porous rotating disk with variable fluid properties," American Journal of Heat and Mass Transfer, vol. 2, no. 3, pp. 165-189, 2015. 


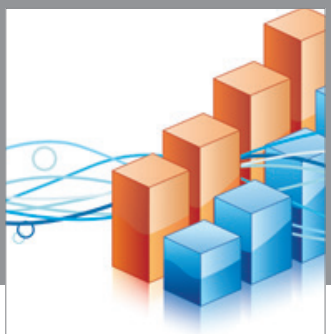

Advances in

Operations Research

vatem alat4

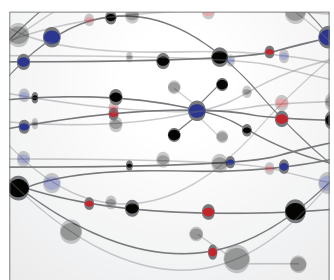

\section{The Scientific} World Journal
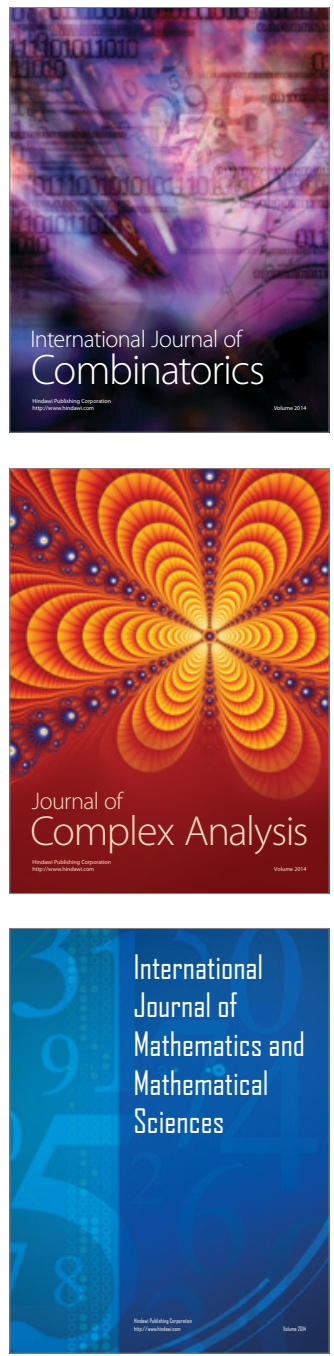
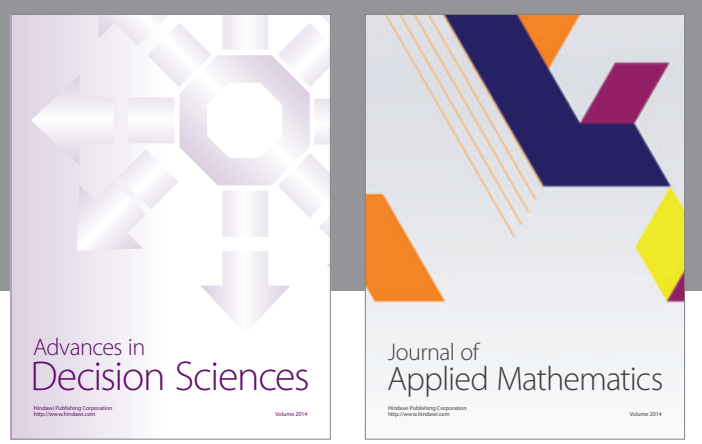

Algebra

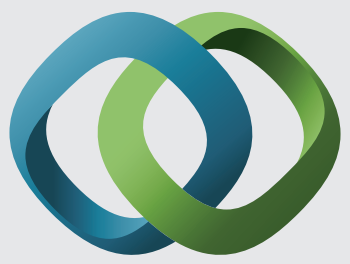

\section{Hindawi}

Submit your manuscripts at

http://www.hindawi.com
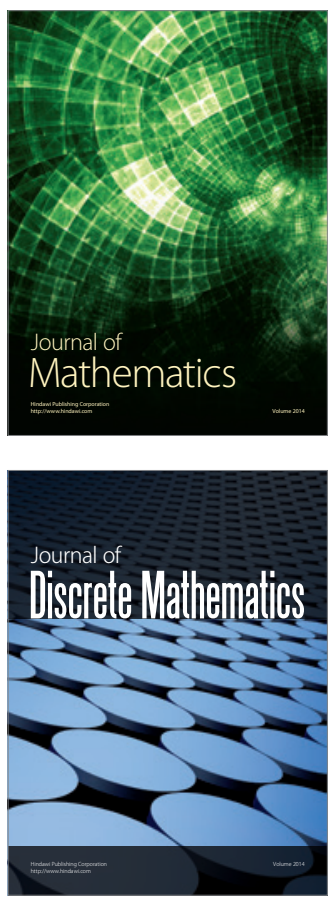

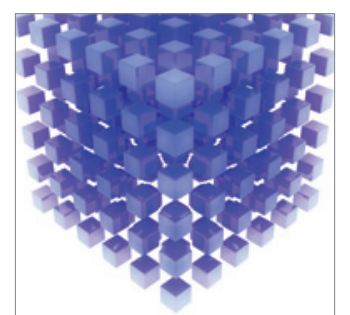

Mathematical Problems in Engineering
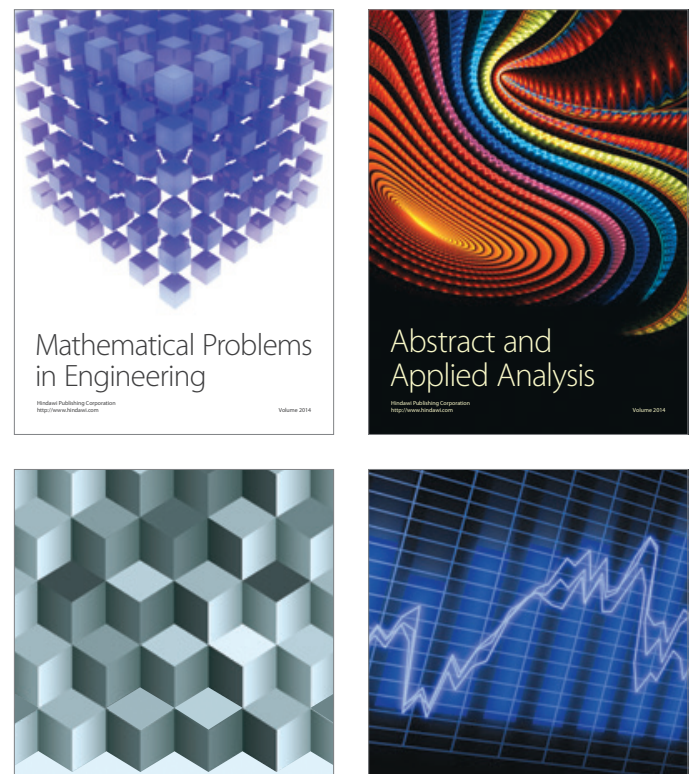

Journal of

Function Spaces

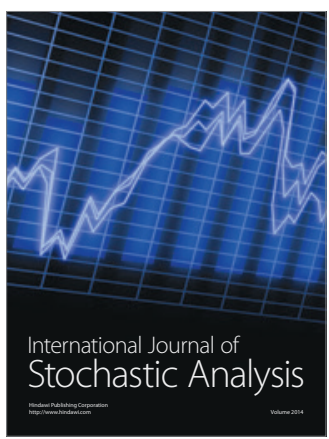

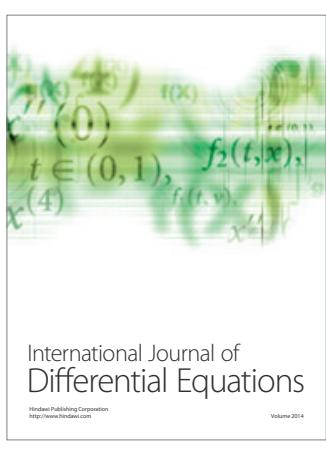
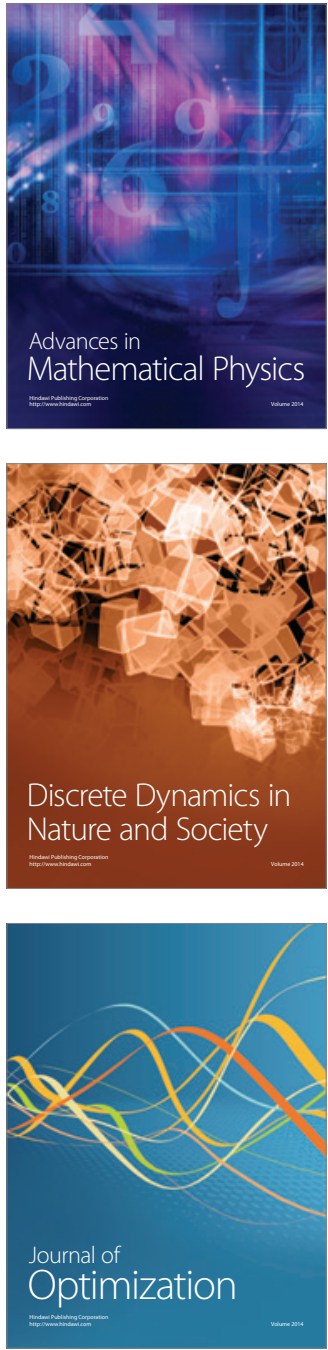